A slightly different version will appear in: Anna Asbury, Jakub Dotlaèil, Berit Gehrke, and Rick Nouwen (eds), The Syntax and Semantics of Spatial P, Amsterdam: Benjamins.

\title{
Silent prepositions: Evidence from free relatives"
}

\author{
IVANO CAPONIGRO \\ University of California, San Diego \\ ivano@ling.ucsd.edu
}

\author{
LISA PEARL \\ University of California, Irvine \\ pearl@uci.edu
}

\begin{abstract}
Silent prepositions are hypothesized to be prepositions that are phonologically null, but syntactically and semantically contentful. Their existence in the grammar has been argued for on the basis of the behavior of expressions like there, now, and that way, which look like nominals but can (or must) behave as prepositional phrases. We bring further evidence in favor of silent prepositions by studying the syntactic and semantic behavior of free relative clauses introduced by the wh-words where, when, and how. We propose a fully compositional syntactic/semantics analysis for these free relatives that accounts for their puzzling nominal/prepositional distributional and interpretative properties. This analysis is crucially based on silent prepositions. We also briefly discuss how our proposal can be extended to interrogative clauses introduced by the same wh-words, as well as what the licensing conditions and semantic properties of silent prepositions are.
\end{abstract}

\footnotetext{
${ }^{*}$ We are very grateful to Grant Goodall, Richard Larson, Howard Lasnik, Carson Schütze, and the participants at the Syntax and Semantics of Spatial P Workshop at the University of Utrecht for their very helpful comments and suggestions. Any remaining errors are, of course, our own.
} 


\section{Introduction: Silent prepositions}

As noticed by Emonds $(1976,1987)$, there are a restricted number of phrases in English that look like NPs, but have the same distribution and interpretation as either NPs or PPs. Larson (1985) labels these phrases bare NP-adverbs, while McCawley (1988) calls them adverbial NPs. An example is the string few places that I cared for, which appears to simply be a (complex) NP. In (1)a, it occurs in a position in which only NPs usually occur (i.e. the subject position of a predicate like be beautiful), receiving the usual interpretation of an NP subject (i.e. an individual or a generalized quantifier). However, in (1)b the very same string occurs in the same position as the bracketed locative PP adjunct in (1)c and has the same interpretation as that PP.

(1) a. [Few places that I cared for] are really beautiful. NP-like

b. You have lived [few places that I cared for]. PP-like

c. You have lived [PP in [few places that I cared for]].

Adverbial NPs are restricted to three semantic areas: spatial expressions (2), temporal expressions (3), and manner expressions (4). ${ }^{1}$

(2) Spatial adverbial NPS
a. You have lived [there]. PP-like
b. [There] is really beautiful. NP-like
c. I went [home]. $\quad P P$-like
d. [Home] never changes. NP-like

(3) Temporal adverbial NPs

a. John arrived [that day]/[Sunday]/[yesterday]. PP-like

b. [That day]/[Sunday]/[Yesterday] was fantastic. NP-like

(4) Manner adverbial NPs

a. You pronounced my name [that way]/[every way one could imagine]. PP-like

b. [That way]/[Every way one could imagine] was not feasible. NP-like

NPs from other semantic areas do not exhibit the same behavior; they can only behave like NPs and not like PPs (5).

\footnotetext{
${ }^{1}$ Examples (2)a, (3)a, and (4)a from Larson (1985).
} 
(5) a.*Jack came $[$ her $] /[$ Lily $] /[$ the person he is in love with]. $\quad * P P$-like (cf. Jack came for/with/after [her]/[Lily]/[the person he is in love with].)

b. [She]/[Lily]/[the person he is love with] does not really like him. NP-like

Larson (1985) argues that adverbial NPs are syntactically NPs with the (lexical) property of self-assigning Case, but Emonds (1987) and McCawley (1988) convincingly show that Larson's proposal is empirically and theoretically problematic. Emonds $(1976,1987)$ and McCawley (1988) argue that adverbial NPs are NPs that can occur in positions to which no Case is assigned and behave like PPs in virtue of a silent preposition that takes the NP as its complement (6).

(6)

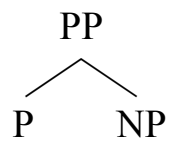

\section{silent $P$ adverbial NP}

In this paper, we bring further support for silent prepositions in the grammar by looking at the syntactic and semantic behavior of embedded non-interrogative wh-clauses known as free relatives (FRs). The bracketed FR in (7)a has the same distribution and roughly the same interpretation as the NP in (7)a'. Yet, in (7)b what looks like the very same FR now occurs in the same position as the PP in (7)b' and receives a similar interpretation.
a. [Where you used to live] was really great.
NP-like
a'. [NP The little town you used to live in] was really great.
b. I live [where you used to live].
PP-like

b'. I live [pp in [the town you used to live in]].

We will argue that this and another puzzling property of FRs can receive a straightforward account if silent Ps are available in the grammar and the same structure as in (6) is assumed. The only difference is that the silent P takes a FR as its complement rather than an adverbial NP (8). ${ }^{2}$

(8)

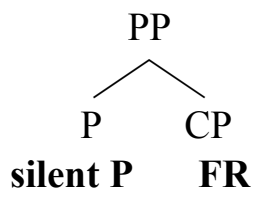

The paper is organized as follows. First, we will discuss two puzzles about FRs (§2), and present a proposal that accounts for them $(\S 3)$. Then, we will bring further independent evidence to support the proposal $(\S 4)$, and discuss potential alternatives and their associated

\footnotetext{
${ }^{2}$ We will discuss later why we assume that a FR is syntactically a CP.
} 
problems (§5). Finally, we will briefly mention some open issues and conclude (§6).

\section{Two puzzles about free relatives}

We focus on two puzzles (among many) which FRs raise that have not received much attention previously. We argue that they can be accounted for by means of silent Ps.

\subsection{Puzzle I: Are FRs like NPs or PPs?}

Some FRs can have the same distribution and interpretation as NPs or PPs. These are the FRs that are introduced by the wh-words where (place), when (time), how (manner) (henceforth, w/w/h FRs). The examples from (9)-(11) illustrate this point. (9)a shows that a w/w/h FR introduced by the spatial wh-word where can behave like an NP, since it can be replaced and paraphrased with the complex NP the place that this very tree grows (9)a'. On the other hand, (9)b shows that what looks like the very same w/w/h FR can syntactically and semantically behave like the complex PP in the place that this very tree grows (9)b'. The same is true for $\mathrm{w} / \mathrm{w} / \mathrm{h}$ FRs introduced by the temporal wh-word when (10) and the manner wh-word how (11).

(9) a. Lily adores [FR where this very tree grows [ $\mathrm{PP} \_$] ].

a'. Lily adores [NP the place that this very tree grows [ $\left.\left.\mathrm{PP}_{-}\right]\right]$.

b. Lily napped [FR where this very tree grows [PP _ ] ].

b'. Lily napped [ $\mathrm{pp}$ in the place that this very tree grows [

(10) a. Lily dreaded [FR when Jack had to go [PP _ ] ].

a'. Lily dreaded [NP the time/moment that Jack had to go [PP _ ]].

b. Lily cried [FR when Jack had to go [PP _ ]].

b'. Lily cried [ $\mathrm{Pp}$ at the time that Jack had to go [

(11) a. Lily loathes [FR how all thieves work [PP _ ] ] - secretly.

a'. Lily loathes [NP the way that all thieves work [

b. Jack works [FR how all thieves work [PP _ ] ] - secretly.

b'. Jack works [ $\mathrm{PP}$ in the way that all thieves work [ $\left.\left.\mathrm{PP}_{\text {_ }}\right]\right]$ - secretly.

Bresnan and Grimshaw (1978: §5) were the first to notice this distribution of w/w/h FRs, though they only discuss examples of FRs introduced by where and when, without mentioning FRs introduced by how.

So, we see that $\mathrm{w} / \mathrm{w} / \mathrm{h}$ FRs exhibit the same puzzling behavior as adverbial NPs: they can have the same distribution and interpretation as either NPs or PPs. 


\subsection{Puzzle II: An NP or a PP gap?}

The second puzzle concerns the nature of the gap within w/w/h FRs. To the best of our knowledge, this has not been noticed before. Similar to other wh-clauses like interrogatives and headed relative clauses, FRs are characterized by a gap in argument or adjunct position. In (9)-(11) above, we marked and labeled the gaps in the w/w/h FRs and the corresponding headed relatives. All the gaps are PP gaps, and this is not by chance. It does not matter whether the whole w/w/h FR behaves like an NP or a PP - only a PP gap is licensed within the $\mathrm{w} / \mathrm{w} / \mathrm{h}$ FR. Even if the predicate in the w/w/h FR selects for an NP, w/w/h FRs can not yet license an NP gap - and it does not matter if the whole w/w/h FR behaves like a PP (12)a or an NP (13)a. Notice that the corresponding headed relatives can license an NP gap, independently from the properties of their heads (cf. (12)a vs.(12)b and (13)a vs. (13)b).

(12) a. ?* Lily always naps [FR where/when/how Jack despises [NP _ ]].

b. Lily always naps [PP $\{$ in the place $\} /\{$ at the time $\} /\{$ in the way $\}$ that Jack despises $\left[\mathrm{NP} \_\right]$].

(13) a. ?* Lily adores [FR where/when/how Jack despises [NP _ ]].

b. Lily adores [NP $\{$ the place $\} /\{$ the time $\} /\{$ the way $\}$ that Jack despises [NP _ ] ].

There is an important exception, though. An NP gap can be licensed as the complement of an overt $\mathrm{P}$ in a w/w/h FR that is introduced by where, whether the whole FR behaves like an NP or a PP (in $\S 4.1$, we will discuss similar examples with FRs introduced by the wh-word when). For example, the w/w/h FR introduced by where in (14)a allows for an NP gap in the complement position of the P past. Since past can only take NP complements, we can be sure that we are dealing with an NP gap. The whole FR behaves like an NP. In fact, it can be replaced and paraphrased with the bracketed complex NP in (14)b. Similarly, the w/w/h FR in (15)a licenses an NP in the complement position of the preposition through, though it behaves like a PP, as shown in (15)b.

(14) a. Jack disliked [FR where we just ran [PP [P past] [NP _ ] ] - it smelled funny.

b. Jack disliked [NP the place we just ran [PP [P past] [NP _ ] ] ] - it smelled funny.

(15) a. Lily lives [ $\mathrm{FR}$ where we have to fly [PP [P through] [NP _ ] ] on our way to Vancouver].

b. Lily lives [PP in the area we have to fly [PP [P through] [NP _ ] $]$ on our way to Vancouver].

To sum up, the second puzzle about w/w/h FRs we will address is that only a PP gap seems to be licensed inside $\mathrm{w} / \mathrm{w} / \mathrm{h}$ FRs, unless an overt $\mathrm{P}$ occurs, which then triggers the licensing of an NP gap as its complement. 


\section{Proposal}

The two puzzles just discussed can be solved by giving a syntactic and semantic analysis of $\mathrm{w} / \mathrm{w} / \mathrm{h}$ FRs that relies on silent Ps. Let us start with a preliminary syntactic assumption: FRs introduced by bare phrasal ${ }^{3}$ wh-words (who, what, where, when, how) are plain CPs with a moved wh-phrase in their specifiers. This is just for the sake of simplicity, since the syntax of FRs is an open issue that goes largely beyond the purposes of this paper. ${ }^{4}$ Nonetheless, nothing crucial in our proposal hinges on this assumption. Any analysis in which the wh-word in a FR is base-generated in the gap position and moved to a higher position will suffice.

Our proposal then can be articulated in two main claims. First, a whole w/w/h FR (i.e. a $\mathrm{CP}$ ) can occur as the complement of a silent P. When the w/w/h FR is the complement of a silent $\mathrm{P}$, the whole $\mathrm{w} / \mathrm{w} / \mathrm{h}$ FR looks as if it is a PP. Note that this is because the $\mathrm{w} / \mathrm{w} / \mathrm{h}$ FR is embedded within an actual PP, whose head is silent (16)a. When the w/w/h FR is not the complement of a silent $\mathrm{P}$, then it behaves like an NP, just as all other FRs introduced by bare phrasal wh-words do (16)b. This accounts for the first puzzle, where w/w/h FRs behave sometimes as NPs and sometimes as PPs.

(16) a.

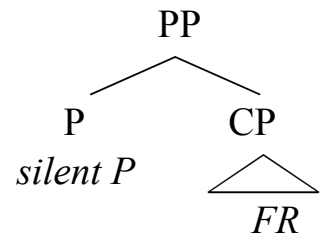

b.

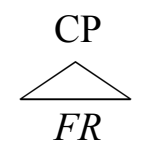

Our second claim is that the wh-words where, when, and how, which introduce $\mathrm{w} / \mathrm{w} / \mathrm{h}$ FRs, are syntactically and semantically NPs that can only be base-generated as the complement of a possibly silent P (17).

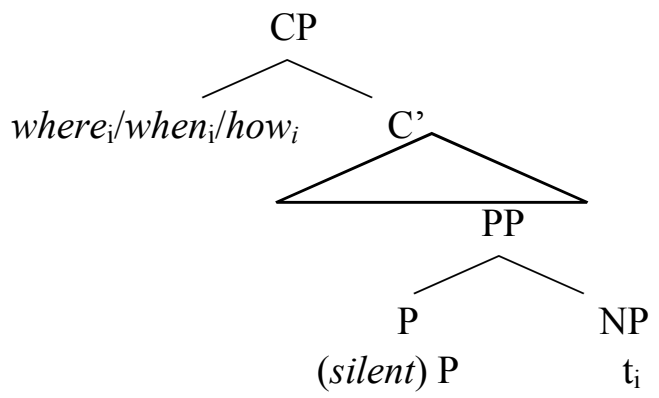

3 "Bare" excludes FRs introduced by wh-ever (whoever, whatever, wherever, whenever), "phrasal" excludes FRs introduced by wh(-ever) + XP (e.g. I'll drink what(ever) beer you drink).

${ }^{4}$ See Grosu (2003) and van Riemsdijk (2005) for an overview of the open issues of the syntax of FRs, the proposals have been made, and their problems. 
The wh-word that is base-generated as the sister of $\mathrm{P}$ moves to the Spec of CP for independent reasons, specifically whatever the reasons for wh-movement in English are. The wh-word leaves behind an NP trace, which is an NP gap. Note that this NP gap looks identical to a PP gap when the P is silent. If the P is overt as in (14) and (15) above, it becomes clear that at least where is an NP. Also, according to our analysis, silent Ps are always stranded. This is due to independent semantic reasons that will be discussed below. This accounts for the second puzzle, in which FRs seem to license PP gaps and NP gaps.

Let us now look at the detailed syntactic derivations of two of the previous examples in order to make concrete how our proposal works. The syntactic tree in (18)b includes the CP of a w/w/h FR that behaves like an NP, since it occurs in the complement position of a predicate like adores (which selects for an NP complement). The $\mathrm{w} / \mathrm{w} / \mathrm{h}$ FR contains a PP with a silent $\mathrm{P}$ and a wh-trace as its complement, resulting from the wh-movement of where to the Spec of CP of the $\mathrm{w} / \mathrm{w} / \mathrm{h}$ FR. Notice that the fact that $\mathrm{P}$ is silent makes the NP gap perceptually indistinguishable from a true PP gap like in the wh-interrogative clause In which areas does this tree grow $\left[\mathrm{PP}_{-}\right]$?

(18) a. Lily adores [FR where this very tree grows].

b.

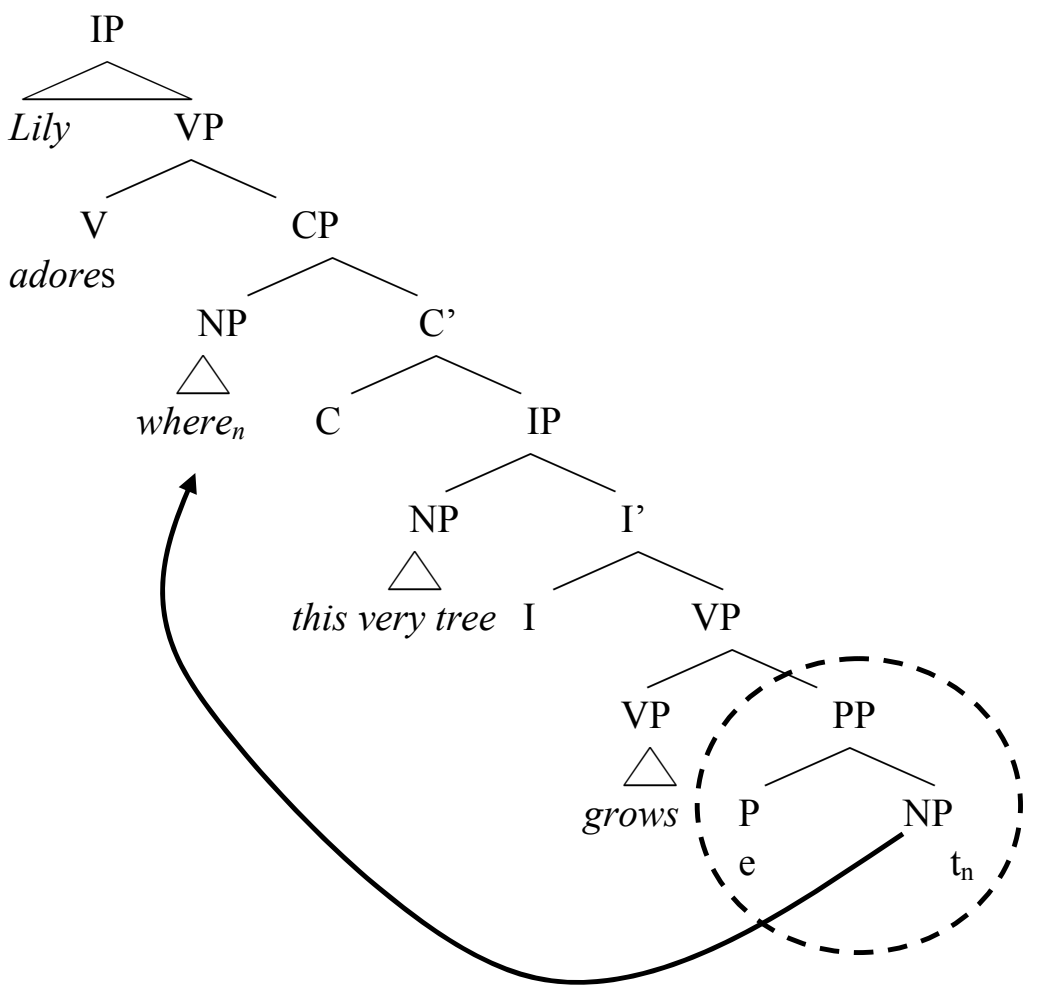

The next example (19)a contains the same w/w/h FR as in (18), but this time the $\mathrm{w} / \mathrm{w} / \mathrm{h}$ FR specifically behaves like a PP. In fact, the FR looks as if it was occurring where a PP, but not an NP, can occur, that is as an adjunct of the predicate napped. But our tree in (19)b shows that this apparent puzzling behavior can once again be accounted for as a perceptual illusion. The $\mathrm{CP}$ of the $\mathrm{w} / \mathrm{w} / \mathrm{h}$ FR occurs in the complement position of a silent preposition $\mathrm{P}_{2}$. Therefore, the whole w/w/h FR still behaves like an NP, as in (18)b . However, $\mathrm{PP}_{2}$, which is 
made of its head $\mathrm{P}_{2}$ and the $\mathrm{CP}$ of the $\mathrm{w} / \mathrm{w} / \mathrm{h} \mathrm{FR}$ as its complement, is perceptually indistinguishable from just the $\mathrm{CP}$ of the $\mathrm{w} / \mathrm{w} / \mathrm{h} \mathrm{FR}$, since $\mathrm{P}_{2}$ is silent. The internal structure of the w/w/h FR with the silent preposition $\mathrm{P}_{1}$ in (19)b is identical to (18)b above.

(19) a. Lily napped [FR where this very tree grows].

b.

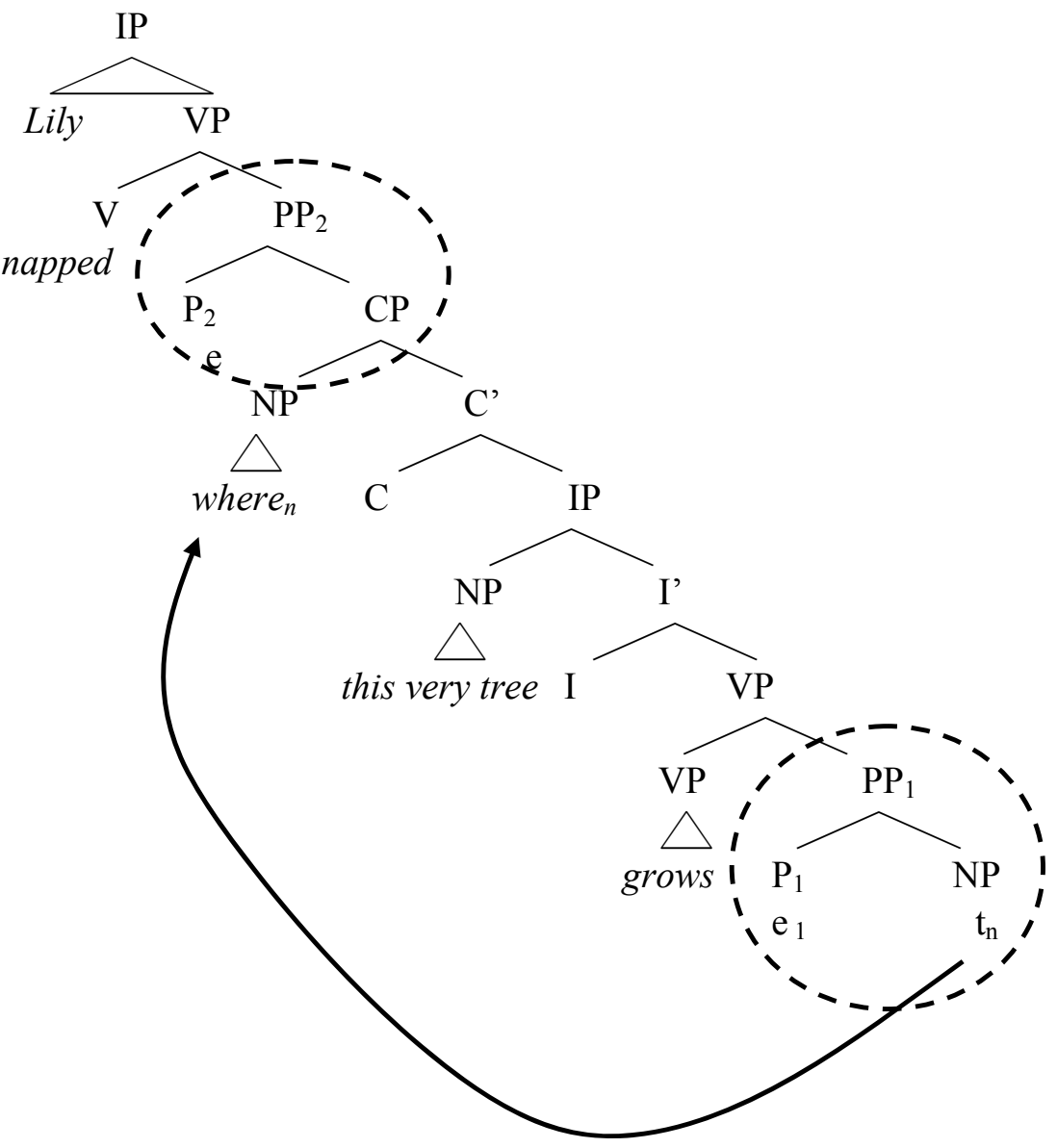

Let us now move to the semantics of w/w/h FRs. This is relevant for our purposes for at least two reasons. First, we want to make sure that the syntactic analysis just suggested is compatible with a semantic analysis that captures the semantic intuitions we discussed earlier: w/w/h FRs should be semantically equivalent to either NPs or PPs. Second, we need a detailed semantics for $\mathrm{w} / \mathrm{w} / \mathrm{h}$ FRs, since our argument to support obligatory stranding of silent Ps is crucially based on the semantic derivation.

Following the semantic analysis for FRs in Caponigro (2004), we pursue the idea that w/w/h FRs denote an individual, like non-quantified NPs. How do w/w/h FRs end up denoting an individual? Let us go step by step from the bottom up, following the schema in (20). 


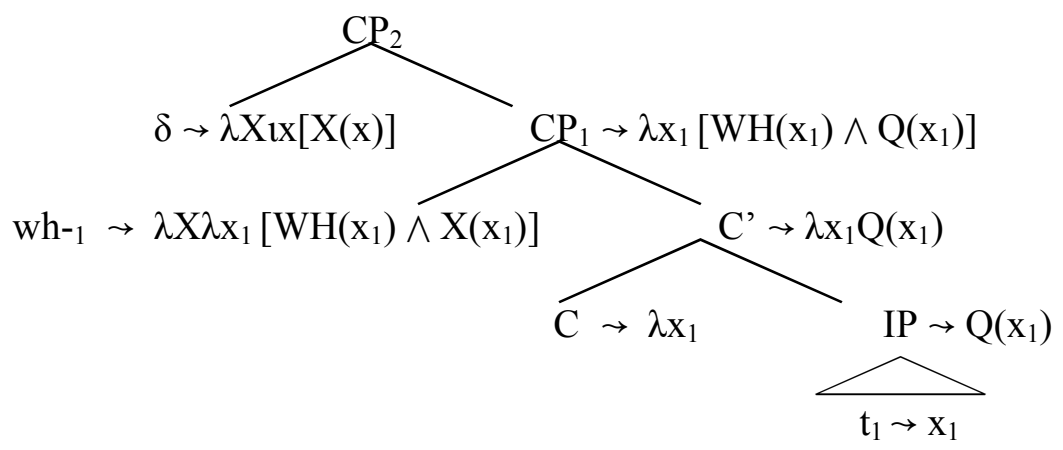

The wh-trace within IP translates into a variable that is bound at by the set-formation operator, the lambda operator (which we assuming to be under $\mathrm{C}$ just for the sake of simplicity). Therefore, C' denotes the set of individuals that have the property Q (i.e. the set $\left.\lambda \mathrm{x}_{1} \mathrm{Q}\left(\mathrm{x}_{1}\right)\right)$ or, equivalently, the set of individuals that make the proposition $\mathrm{Q}(\mathrm{x})$ expressed by the IP true.

The next step is to combine this set with the semantic contribution of the wh-word. But what is the semantic contribution of where, when, or how? These words behave like set restrictors: they apply to a set of individuals and return a subset. In particular, where applies to a set of individuals and returns all and only the individuals that are locations, when all and only instants or situations, and how all and only manners. When one of these wh-words applies to the set of individuals $\lambda \mathrm{x}_{1} \mathrm{Q}\left(\mathrm{x}_{1}\right)$, it returns the subset $\lambda \mathrm{x}_{1}\left[\mathrm{WH}\left(\mathrm{x}_{1}\right) \wedge \mathrm{Q}\left(\mathrm{x}_{1}\right)\right]$, i.e. the set of individuals that have both the property $\mathrm{Q}$ and the property $\mathrm{WH}$, the restriction conveyed by the wh-word (see Caponigro 2004 for further details and arguments).

So at the level of $\mathrm{CP}_{1}$, w/w/h FRs denote the set of locations, instants/situations, or manners that satisfy the property Q. But, according to our syntactic analysis, w/w/h FRs, which so far denote sets of individuals (semantic type: $<\mathrm{e}, \mathrm{t}>$ ), occur in the argument positions of heads (verbs or preposition) that select for an individual-denoting expressions (semantic type: <e>). It appears we have a so-called type mismatch. Partee (1986), Chierchia (1998), and Dayal (2004) have argued that type-shifting rules are made available by the grammar to fix type-mismatches. Among those, iota ( $l$ ) applies to a set $\mathrm{P}$ and returns its maximal individual (21).

$$
\operatorname{iota}(\mathrm{l}): \mathrm{P} \rightarrow \operatorname{cxP}(\mathrm{x})(<\mathrm{e}, \mathrm{t}>\rightarrow<\mathrm{e}>)
$$

The empirical evidence for a type-shifting rule like iota originally comes from the crosslinguistic behavior of nominals, in particular bare plurals and bare singulars (Chierchia, 1998; Dayal, 2004). Caponigro (2004) - developing a suggestions in Jacobson (1995) - adds further support coming from FRs. He syntactically encodes iota by means of a silent operator $\delta$, adjoined to $\mathrm{CP}$ of FRs. We did the same in (20). The set of individuals denoted by $\mathrm{CP}_{1}$ in (20) type-shifts to its maximal (plural) individual when it combines with $\delta$ at the level of the adjoined $\mathrm{CP}_{2}$.

In (22) and (23) below, we repeat the syntactic trees in (18) and (19) respectively and add their logical translations in accordance to the analysis we just proposed. PPs are treated as extra arguments of the predicate they modify for sake of simplicity. Also, no detailed semantic analysis is given for PPs, since it is not relevant for our purposes. Any semantics for 
Ps would suffice as long as Ps select for an individual-denoting complement. Finally, we ignore the deictic nature of nominal this very tree for the sake of simplicity and we translate it as the simple individual-denoting expression $t v t$.
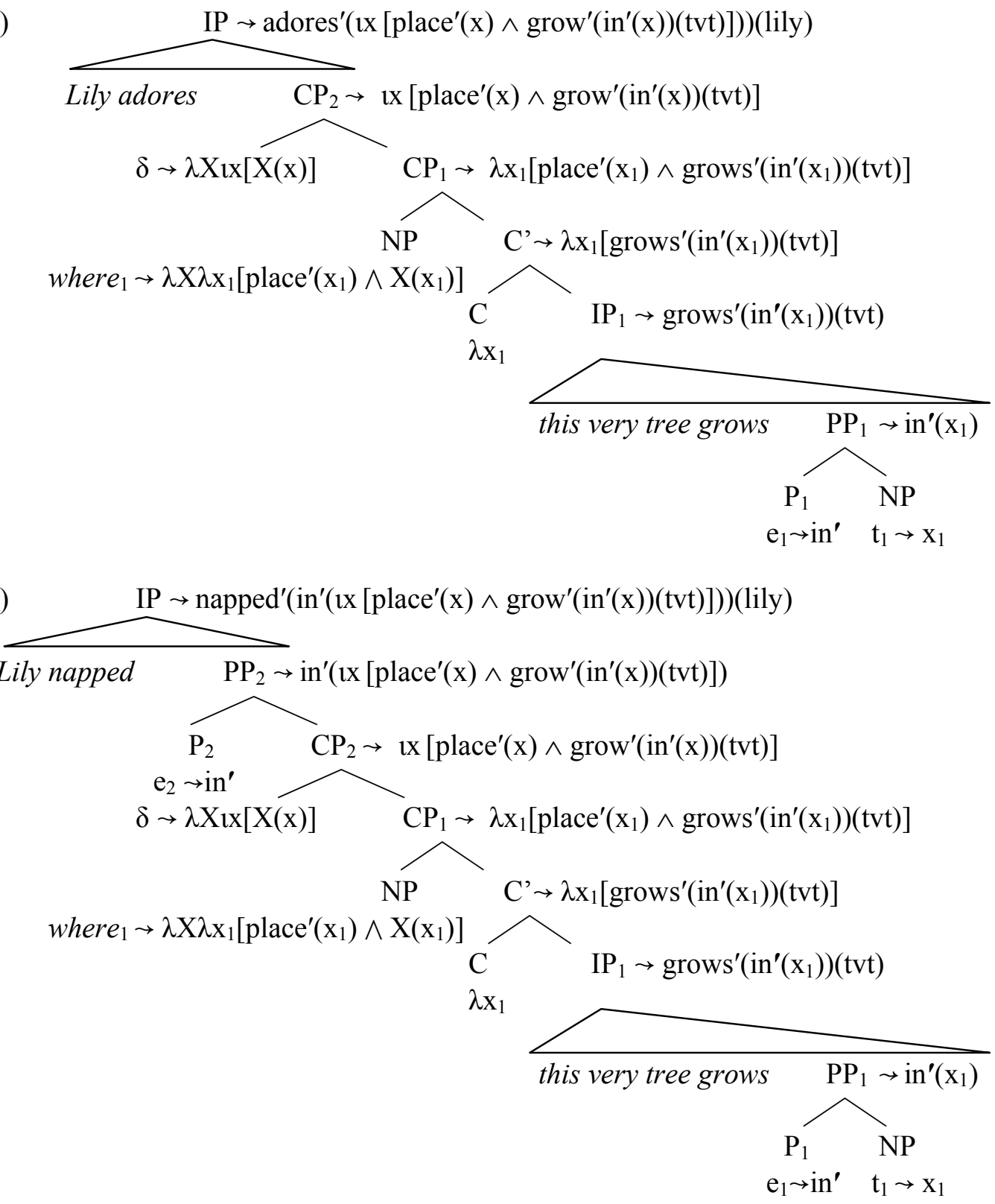

To sum up, we have argued that w/w/h FRs are very similar both syntactically and semantically to the FRs introduced by the bare phrasal wh-words who and what. Syntactically, they are wh-CPs (or any other phrasal structure that would better account for the property of FRs); semantically, they denote an individual. The crucial difference is the structure of the wh-words that introduce w/w/h FRs: where, when, and how are always base-generated as the complement of a possibly silent $\mathrm{P}$. In addition, whenever the $\mathrm{w} / \mathrm{w} / \mathrm{h} \mathrm{FR}$ 
behaves as if it was a PP, the whole w/w/h FR itself is the complement of another silent P.

\section{Further evidence}

In this section, we present further evidence in favor of our analysis. We show that silent Ps either within a w/w/h FR or as its sister - can be replaced with overtly realized Ps in some cases $(\S 4.1)$. We give further support to our proposal that where, when, and how are NPs, rather than PPs, by showing that Ps usually take an NP as their complement, rather than a PP (§4.2). We then show that our proposal about the syntactic and semantic nature of $\mathrm{w} / \mathrm{w} / \mathrm{h}$ FRs has larger empirical coverage by extending it to wh-interrogatives that are introduced by where, when or how (§4.3). Finally, we show that the same kinds of restrictions on licensing silent Ps that we noticed for adverbial NPs earlier $(\S 1)$ are found with w/w/h FRs as well (§4.4), which supports the claim that these two constructions are similar and should receive a similar analysis.

\subsection{Overt Ps}

We have argued that the wh-trace within a w/w/h FR is always the complement of a possibly silent $\mathrm{P}$ and that the whole $\mathrm{w} / \mathrm{w} / \mathrm{h}$ FR is the complement of another silent $\mathrm{P}$ whenever the $\mathrm{w} / \mathrm{w} / \mathrm{h}$ FR behaves as if it was a PP. Though these two Ps are silent most of the time, there are cases where one or both of them are overt. In (24), we see that the P that takes the wh-trace of where as its complement can be overtly realized, in this case as the P past. In (25), we see that the $\mathrm{P}$ that takes the whole $\mathrm{w} / \mathrm{w} / \mathrm{h}$ FR introduced by where as its complement can be overtly realized, in this case as the $\mathrm{P}$ near. In (26), both the $\mathrm{P}$ sister to the wh-trace and the $\mathrm{P}$ sister to the w/w/h FR are overtly realized as through and near, respectively.

(24) Jack disliked [FR where $\mathrm{m}_{\mathrm{m}}$ we just ran $\left[\mathrm{PP}[\mathrm{P}\right.$ past $\left.\left.]\left[\mathrm{NP} \mathrm{t}_{\mathrm{m}}\right]\right]\right]$ - it smelled funny.

(25) Lily lives [PP1 [P1 near] [FR where ${ }_{\mathrm{m}}$ we had dinner [PP2 [P2 $\left.\left.\mathrm{e}\right]\left[\mathrm{NP} \mathrm{t}_{\mathrm{m}}\right]\right]$ last night]].

(26) Lily lives [PP1 [P1 near] [FR where ${ }_{\mathrm{m}}$ we have to fly [PP2 [P2 through] $\left.\left[\mathrm{NP} \mathrm{t}_{\mathrm{m}}\right]\right]$ on our way to Vancouver]].

In (27), we see that w/w/h FRs introduced by the wh-word when can also occur as the complement of over Ps like from or to, while (28) shows that the trace of the wh-word when can be the complement of an overt $\mathrm{P}$ as by.

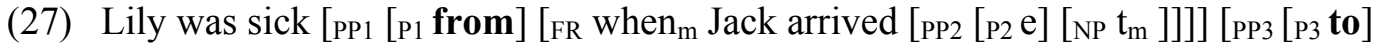
$\left[\mathrm{FR}\right.$ when $_{\mathrm{p}}$ he left [PP4 [P4 e] [NP $\left.\left.\left.\left.\mathrm{t}_{\mathrm{p}}\right]\right]\right]\right]$.

(28) Lily's schedule can't accommodate [ when $_{\mathrm{m}}$ Jack needs the car [PP [P by [NP $\left.\left.\left.t_{\mathrm{m}}\right]\right]\right]$. 
Finally, (29) shows that a whole FR introduced by the wh-word how can occur as the complement of an overt preposition like $o n .^{5}$

(29) Lily knew that Jack was about to get upset based [PP1 [P1 on] [how $\mathbf{h}_{\mathrm{m}}$ he was looking at her [ $\left.\left.\left[\mathrm{PP} 2[\mathrm{P} 2 \mathrm{e}]\left[\mathrm{NP} \mathrm{t}_{\mathrm{m}}\right]\right]\right]\right]$

We take these cases where the $\mathrm{P}$ is overt as further evidence in favor of the existence of the two distinct $\mathrm{P}$ heads we are arguing for, even when these $\mathrm{P}$ heads are not phonologically realized.

\subsection{Complements of $P$ must be NPs}

We just saw that where, when, and how can be base-generated as complement of Ps like past, through, or near. These Ps can take an NP complement, but not a PP one, as shown in (30)-(32).

(30) a. past [NP the house]

b. *past [pp at the house]

(31) a. through [NP the grass]

b. *through [PP on the grass]

(32) a. near [NP the store]

b. * near [pp in the store]

Since where, when, and how can be base-generated as the complement of Ps like past, through, or near it follows that they must be NPs, rather than PPs. We take this as independent evidence in favor of our conclusions that the categorical status of where, when, and how is NP, rather than PP.

\footnotetext{
${ }^{5}$ We have not been able to find any example in which the trace of how can occur as the sister of an overt preposition. Although we do not have an account for this asymmetry of how with respect to where and when, we note two possibly related facts. First, a headed relative clause with the nominal way as its head, which is the most natural paraphrase for a FR introduced by how, can be optionally followed by the complementizer that or, crucially, by the relative pronoun which preceded by the preposition in. No other preposition can occur between way and which.

(i) Lily knew that Jack was about to get upset based [[on] [the way [(that)/(in/*by/*with which) he was looking at her]]].

Second, the $\mathrm{P}$ in can never occur as the sister of the trace of where, when, and how. If for whatever reason in is the only overt preposition in English that is compatible with very general manner expressions like way, but at the same time is incompatible with where, when and how, it follows that no overt preposition can ever take the trace of how as its complement.
} 


\subsection{Wh-interrogative clauses}

Our claim that where, when, and how are always base-generated as the complement of a $\mathrm{P}$ would look suspiciously restricted if it was true just for w/w/h FRs rather than for any wh-clause introduced by those wh-words. Wh-interrogatives introduced by where behave exactly like the w/w/h FRs we saw in $\S 4.1$ (33)-(34). Their wh-word is base-generated as the complement of a $\mathrm{P}$ that can also be overt. Huang (1982:536) makes a similar point.

(33) Where $\mathrm{m}_{\mathrm{m}}$ did we just run $\left[\mathrm{PP}[\mathrm{p}\right.$ past $\left.]\left[\mathrm{NP}_{\mathrm{m}}\right]\right]$ ?

(34) Where ${ }_{\mathrm{m}}$ do we have to fly $\left[\mathrm{PP}[\mathrm{P}\right.$ through $\left.]\left[\mathrm{NP}_{\mathrm{m}}\right]\right]$ on our way to Vancouver?

Also, if no overt P is present, wh-interrogatives introduced by where, when, and how only license what looks like a PP gap on the surface (35). This is the same behavior that we observed in w/w/h FRs (§2.2).

(35) a. *Where/when/how did Lily despise [NP _ _ ]?

b. Where/when/how did Lily sleep [ ${ }_{\mathrm{PP}}$ ] $]$ ?

\subsection{Semantic restrictions on silent P licensing and adverbial NPs}

At the very beginning of this paper $(\S 1)$, we mentioned that adverbial NPs in English look like NPs, but can behave like NPs or PPs. We also noticed adverbial NPs are restricted to three semantic areas: spatial expressions (36), temporal expressions (37), or manner expressions (38). NPs from other semantic areas do not exhibit the same pattern (39).

(36) Spatial adverbial NPS
a. You have lived [there]. $\quad$ PP-like
b. [There] is really beautiful. NP-like
c. I went [home]. $\quad$ PP-like
d. [Home] never changes. NP-like

(37) Temporal adverbial NPs

a. John arrived [that day]/[Sunday]/[yesterday]. PP-like

b. [That day]/[Sunday]/[Yesterday] was fantastic. NP-like

(38) Manner adverbial NPS

a. You pronounced my name [that way]/[every way one could imagine]. PP-like

b. [That way $] /[$ Every way one could imagine] was not feasible. NP-like

(39) a. *Jack came [her]/[Lily]/[the person he is in love with].

*PP-like (cf. Jack came for/with/after [her]/[Lily]/[the person he is in love with].)

b. $[\mathrm{She}] /[\mathrm{Lily}] /[$ the person he is love with] does not really like him. NP-like 
W/w/h FRs show interesting similarities with adverbial PPs. As we have seen, w/w/h FRs can also behave like NPs or PPs. In addition, w/w/h FRs are introduced by wh-words that carry the same semantic feature as adverbial NPs: location/space (where), time/situation (when), or manner (how). This contrasts with FRs introduced by other bare phrasal wh-words like who (animate) and what (inanimate) that do not behave like adverbial NPs. In particular, they can occur where NPs can occur, but cannot occur where PPs can occur. As an example, (40)a shows that a FR introduced by what can occur as the complement of a predicate like adore, which selects for an NP complement, as shown (40)a'. However, if the very same FR occurs as the adjunct of an intransitive predicate like work in which only PPs are allowed, as shown in (40)b', then the result is unacceptable, as shown in (40)b. The examples in (41) make a similar point for FRs introduced by who.

(40) a. Lily adores [FR what Jack despises].

NP-like

a'. Lily adores [NP the things Jack despises].

b. *Lily works [FR what Jack despises]. $\quad *$ PP-like

b'. Lily works [PP [P on] [NP the things Jack despises]].

(41) a. Lily won't marry [FR who the king chooses].

NP-like

a'. Lily won't marry [NP the person the king chooses].

b. * Lily will dance [FR who the king chooses].

*PP-like

b'. Lily will dance [PP [P with] [NP the person the king chooses]].

We take these similarities between $\mathrm{w} / \mathrm{w} / \mathrm{h}$ FRs and adverbial NPs as an indication that a similar syntactic and semantic analysis for both constructions is justified. As we briefly mentioned in $\S 1$, an analysis based on silent Ps has been convincingly put forth for adverbial NPs (Emonds, 1976, 1987; McCawley, 1988). We take this to further support our analysis of $\mathrm{w} / \mathrm{w} / \mathrm{h}$ FRs based on silent Ps. In particular, FRs introduced by who and what lack any spatial, temporal, or manner feature; therefore, they cannot occur as the complement of a silent $\mathrm{P}$.

The reason that FRs introduced by who and what lack the relevant features is because the wh-words that introduce them and act as a set restrictor lack them as well. This makes the prediction that who and what should never be base-generated as the complement position of a silent P or, equivalently, FRs introduced by who or what should never license a PP gap. This prediction is borne out. A FR introduced by who or what can license an NP gap in the complement of the P to (42)a, but cannot license a PP gap within what looks like the same FR except for the omitted P (42)b. As expected, a FR introduced by where can easily license a PP gap in the very same environment (42)c.

(42) a. Lily doesn't like [FR who/what Jack goes to [NP _ ] every Friday night].

b. * Lily doesn't like [FR who/what Jack goes [PP __ every Friday night].

c. Lily doesn't like [FR where Jack goes [PP__ every Friday night].

\section{Problems with alternative accounts}

In this section, we discuss potentially alternatives to our proposal and where they are 
problematic.

\subsection{Against an ambiguity account}

A simple alternative to our proposal could be to assume that where, when, and how are syntactically ambiguous: they are listed in the lexicon as both NPs and PPs. There are two issues with this approach, however. First, we would not be dealing with the idiosyncratic behavior of just one word, but with three lexical items that are members of the same class (wh-words) and exhibit very similar syntactic behaviors (introduce wh-clauses, license both NPs and PP gaps, etc.). Also, we have observed the same pattern in other languages with $\mathrm{w} / \mathrm{w} / \mathrm{h}$ FRs like Italian (although a careful crosslinguistic investigation is needed). This kind of (crosslinguistic) systematic ambiguity appears more like restating the generalization than an actual explanation.

Second, an ambiguity approach would end up making at least two incorrect predictions in this specific case. If where, when, and how were listed in the lexicon as both NPs and PPs, then they should be able to license either NP or PP gaps in the clause they introduce. But this is not the case, since they license only PP gaps (\$2.2). Also, obligatory matching between the syntactic category of the gap and the whole FR would be expected. In particular, if a PP gap is licensed within a w/w/h FR, then the whole w/w/h FR should always behave like a PP. This is also not the case. While where, when, or how only license a PP-gap within w/w/h FRs, the whole $\mathrm{w} / \mathrm{w} / \mathrm{h}$ FR can behave either like an NP or PP, depending on the matrix predicate (43).

(43) a. Lily adores [FR [where/when/how] Jack sleeps [PP_ ] ]. NP-like FR

b. Lily sleeps [FR [where/when/how] Jack sleeps [PP_ ] ]. PP-like FR

\subsection{Against a pied-piping analysis}

Another alternative approach could be based on optional pipe-piping of silent Ps. Like ours, it would assume that where, when, and how are always base-generated as the NP complement of a possibly silent P. Unlike ours, it would also assume that whenever the whole $\mathrm{w} / \mathrm{w} / \mathrm{h}$ FR behaves like a PP, this is due to the entire wh-PP moving to the Spec of CP, rather than just the wh-word. In other words, this approach would replace the stranding of the silent $\mathrm{P}$ in the $\mathrm{w} / \mathrm{w} / \mathrm{h}$ FRs with its pied-piping. There would be no need to postulate another silent $\mathrm{P}$, which, according to our proposal, takes the whole w/w/h FR as its complement. In (44), we see the syntactic derivation of the example we discussed in (19) according to the pied-piping approach: it is the whole wh-PP [e where $]_{\mathrm{m}}$ that moves to Spec of CP, leaving behind the PP trace $t_{\mathrm{m}}$. 
(44) a. Lily napped [FR where this very tree grows].

b.

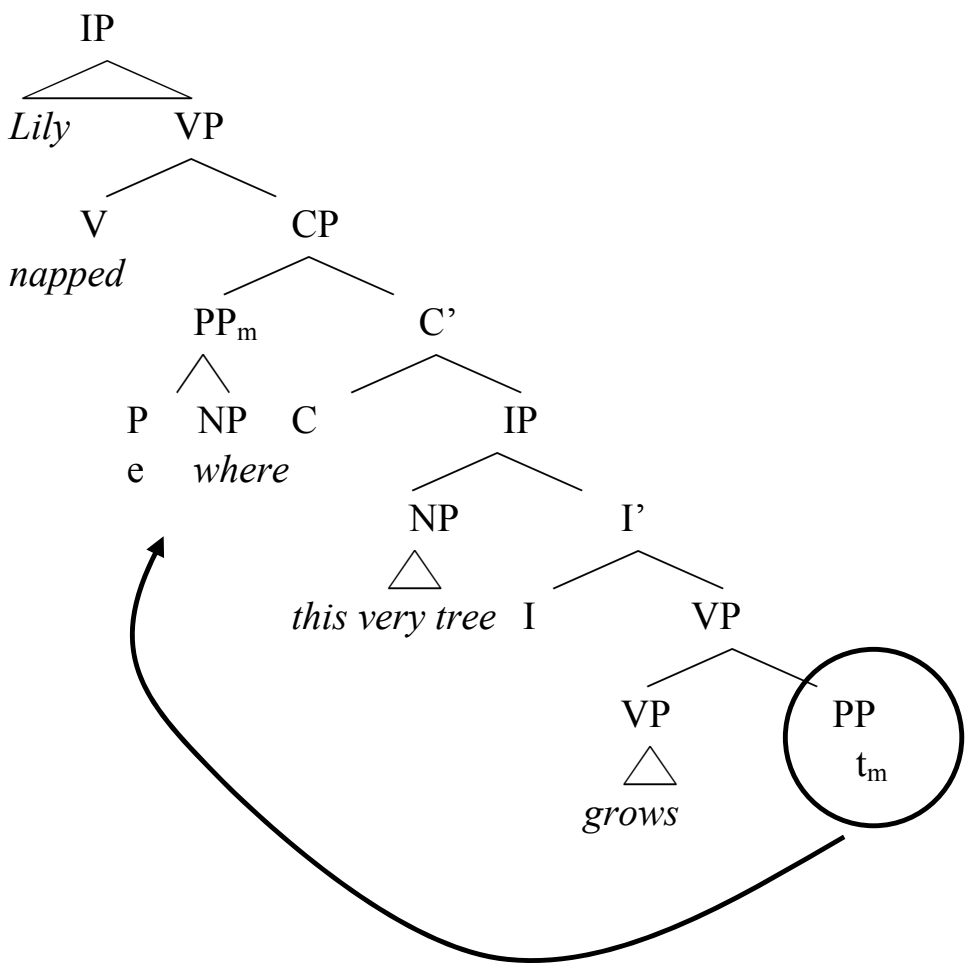

This approach faces at least two major problems. First, it would predict that the silent PP within the w/w/h FR and the whole w/w/h FR should always be interpreted as the same kind of PP, since their interpretation would depend on the very same silent $\mathrm{P}$. This prediction is not borne out. The $\mathrm{w} / \mathrm{w} / \mathrm{h}$ FR in (45)a is interpreted as basically equivalent to the bracketed complex NP in (45)b, the only relevant difference being that all Ps are overt in (45)b. In particular, the PP gap in (45)b results from the movement of the PP at which, which is headed by the overt P at. Yet, the whole complex NP occurs as the complement of a PP that is headed by a different P: to. Crucially, the two Ps are not identical and cannot be interpreted equivalently. If we try to interpret the clauses this way, at would also precede the whole complex NP and the result would be semantically different from the intended meaning of (45)a - as well as unacceptable, as shown by (45)c. Thus, a pied-piping approach with only one silent $\mathrm{P}$ as in (45)d fails. In contrast, our approach can easily handle these facts, since two independent silent Ps would be present in the syntactic/semantic representation, as shown in (45)e. 
(45) a. Lily just went [FR where Jack stayed last year on vacation].

b. Lily just went [PP to [NP the place [PP at which] Jack stayed [PP $\left.\mathrm{t}_{\mathrm{j}}\right]$ last year on vacation]].

c. * Lily just went $\left[\mathrm{PP}\right.$ at [NP the place [PP at which] Jack stayed [ $\left.{ }_{\mathrm{PP}} \mathrm{t}_{\mathrm{j}}\right]$ last year on vacation]].

d. Lily just went $[\mathrm{FR}[\mathrm{PP}[\mathrm{P} \mathbf{e}] \text { [where }]]_{\mathrm{j}}$ Jack stayed [PP $\left.\mathrm{t}_{\mathrm{j}}\right]$ last year on vacation]].

e. Lily just went $\left[\mathrm{PP}\left[\mathrm{P} \mathbf{e}_{\mathbf{1}}\right]\left[\mathrm{FR}[\mathrm{NP} \text { where }]_{\mathrm{j}}\right.\right.$ Jack stayed $\left.{ }_{\mathrm{PP}}\left[{ }_{\mathrm{P}} \mathbf{e}_{2}\right]\left[\mathrm{NP}_{\mathrm{j}}\right]\right]$ last year on vacation]].

A similar point can be made with overt Ps. In (46)a, we see a w/w/h FR with a stranded overt $\mathrm{P}$ to. Notice that to cannot be pied-piped (46)b (at least not overtly) which is already unexpected under a pied-piping approach. Besides that, the w/w/h FR can be paraphrased with a complex NP whose internal $\mathrm{P}$ is to but whose sister is a different P: in/at (46)c. The interpretation that the pied-piping approach would predict is once again unacceptable (46)d and the reason is again the lack of a second $\mathrm{P}$ as a sister of the whole $\mathrm{w} / \mathrm{w} / \mathrm{h}$ FR (46)b. This contrasts with our analysis (46)e.

(46) a. Lily lives [FR where $\mathrm{m}_{\mathrm{m}}$ Jack is about to go [PP to [NP $\left.\left.\left.\mathrm{t}_{\mathrm{m}}\right]\right]\right]$.

b. * Lily lives [FR [PP to where $]_{j}$ Jack is about to go [PP $\left.\left.\mathrm{t}_{\mathrm{j}}\right]\right]$.

c. Lily lives [PP in/at [NP the place where ${ }_{\mathrm{m}}$ Jack is about to go [PP to [NP $\left.\left.\mathrm{t}_{\mathrm{m}}\right]\right]$ ].

d. *Lily lives [pP to [NP the place where $\mathrm{m}_{\mathrm{m}}$ Jack is about to go [PP to [NP $\left.t_{\mathrm{m}}\right]$ ]].

e. Lily lives [PP [P e] [FR [NP where $]_{j}$ Jack is about to go [PP [P to] $\left.\left.\left[\mathrm{NP}_{\mathrm{j}} \mathrm{t}_{\mathrm{j}}\right]\right]\right]$.

Finally, even when the w/w/h FR is preceded by an overt $\mathrm{P}$, the overt $\mathrm{P}$ is never pied-piped with the wh-word from within the FR. It is always part of the matrix clause taking the $\mathrm{w} / \mathrm{w} / \mathrm{h} \mathrm{FR}$ as its complement. The sentence in (47)a can only have the bracketing in (47)b, according to which near is base-generated in the matrix clause and does not form a constituent with where. This is the only bracketing that is compatible with the interpretation the sentence receives, which we tried to paraphrase in (47)c. If near had been pied-piped with where $(47) \mathrm{d}$, then we would expect the interpretation of the sentence to be like (47)e, which is not the case.

(47) a. Jack lives near where we had dinner last night.

b. Jack lives [near [FR where we had dinner last night]].

c. Jack lives [near [the place at which we had dinner last night]].

d. Jack lives [FR [near where] we had dinner last night]].

e. Jack lives [near [the place near which we had dinner last night]].

The second incorrect prediction of the pied-piping analysis concerns the semantic derivation that this approach would require. The wh-PP would move to Spec of CP and leave a PP trace in the gap position within the w/w/h FR, rather than an NP trace. As we saw in $\S 3$, an NP-trace is translated into a variable whose value ranges over individuals (semantic objects of type $<\mathrm{e}>$ ). When the set-formation operation over that variable ( $\lambda$ abstraction) applies, it returns a set of individuals (type $<\mathrm{e}, \mathrm{t}>$ ). On the other hand, a PP-trace translates into a variable whose value ranges over more complex semantic objects, functions whose 
semantic type we call $<\mathrm{f}>$ for convenience. When the set-formation operation over a variable of type $<\mathrm{f}>$ applies, it returns a set of PP-functions of type $<\mathrm{f}, \mathrm{t}>$. Iota, the crucial type-shifting operation that turns a set of individuals into its maximal individual (21), cannot apply this time since there is no set of individuals to begin with. Therefore, the whole $\mathrm{w} / \mathrm{w} / \mathrm{h}$ FR would be left denoting a set of PP-functions, while the matrix clause would require an expression denoting what a PP denote, i.e. just a single PP-function. In conclusion, the FR would be expected not to have the same interpretation as a PP and, therefore not even the same distribution. This is contrary to what the data show.

We have argued that silent Ps are always stranded. This is not particularly problematic for a language like English that allows for preposition stranding extensively. But what about those languages (actually, the majority) that have w/w/h FRs but do not allow for preposition stranding (for instance, Italian)? Unfortunately, we are still far from a deep understanding of preposition stranding and why it is so rare across languages. Until then, preposition stranding cannot be used to make a point against or in favor of our proposal. For instance, suppose that the ban on preposition stranding turns out to phonological in nature (maybe due to the clitic-like nature of Ps in many languages). Then, it would not be surprising that silent Ps are always stranded, since they lack any phonological content by definition. ${ }^{6}$

\section{Some open issues and conclusions}

There are a few open issues that we believe are particularly relevant and deserve further investigation. First, we saw that silent Ps are licensed only as sisters of phrases that carry spatial, temporal, or manner semantic features (adverbial NPs, wh-words, or FRs). It would be interesting to better understand the nature of this restriction and give it a principled explanation.

It may turn out that this open issue is related to another: the semantic content of silent Ps. More specifically, the semantic contribution of silent Ps is "minimal": they are always interpreted as semantically equivalent to Ps like $t o$, in, at, or on whose semantic contribution is often fully recoverable from the semantic contribution of the predicate and the complement. Yet, semantic "lightness" and recoverability are necessary but not sufficient conditions for the licensing of silent Ps. For instance, the semantic contribution of the silent Ps in the w/w/h FRs in (48)a and (49)a, and in the wh-interrogative in (50)a is fully recoverable: something very close to the P from, as shown in (48)b, (49)b, and (50)b. ${ }^{7}$ While from seems semantically "light", none of those silent Ps in these examples is licensed.

(48) a. * Lily really does not like [FR where Jack is coming e].

b. Lily really does not like [FR where Jack is coming from].

\footnotetext{
${ }^{6}$ Thanks to Richard Larson for pointing out the issue of obligatory stranding.

${ }^{7}$ Thanks to Grant Goodall for suggesting this kind of example.
} 
(49) a. * Lily is coming [PP $\mathbf{e}$ [FR where Jack is coming from]].

b. Lily is coming [PP from [FR where Jack is coming from]].

(50) a. * Lily wonders [INT where Jack is coming e]?

b. Lily wonders [INT where Jack is coming from]?

To conclude, we have argued in this paper that if silent Ps are assumed in the grammar, we can account not only for adverbial NPs - which had already been suggested - but also for the puzzling syntactic/semantic behavior of a subclass of FRs. In particular, we have argued that a silent $\mathrm{P}$ can take a FR introduced by where, when, or how as its complement and that where, when, and how are always base-generated as the complement of a possibly silent P. Silent Ps have allowed us to account for two puzzling properties of these FRs: their NP/PP-like behavior and the restrictions of the internal gap they license. In addition, silent Ps have allowed us to account for unexpected similarities between these FRs and adverbial NPs. We believe there is strong evidence and good reason to assume silent Ps as a component of the grammar. Continued investigation, especially crosslinguistic, would further illuminate their syntactic and semantic properties. 


\section{REFERENCES}

Bresnan, J. and Grimshaw, J. 1978. The syntax of free relatives in English. Linguistic Inquiry 9.331-391.

Caponigro, I. 2004. The semantic contribution of wh-words and type shifts: Evidence from free relatives crosslinguistically. Proceedings of Semantics and Linguistic Theory (SALT) $X I V$, R. Young (ed.), 38-55. Ithaca, NY: CLC Publications, Cornell University.

Chierchia, G. 1998. Reference to kinds across languages. Natural Language Semantics 6.339-405.

Dayal, V. 2004. Number marking and (in)definiteness in kind terms. Linguistics and Philosophy 27.393-450.

Emonds, J. 1976. A transformational approach to English syntax. New York: Academic Press.

Emonds, J. 1987. The Invisible Category Principle. Linguistic Inquiry 18: 613-632.

Grosu, A. 2003. A unified theory of 'standard' and 'transparent' free relatives. Natural Language \& Linguistic Theory 21: 247-331.

Huang, J. 1982. Logical relations in Chinese and the theory of grammar. PhD dissertation. Massachusetts Institute of Technology, Boston.

Jacobson, P. 1995. On the quantificational force of English free relatives. Quantification in Natural Languages, E. Bach, E. Jelinek, A. Kratzer, and B. Partee (eds), 451-486. Dordrecht: Kluwer.

Larson, R. 1985. Bare-NP adverbs. Linguistic Inquiry 16: 595-621.

McCawley, J. 1988. Adverbial NPs: Bare or clad in see-through the garb? Language 64: 583-590.

Partee, B. 1986. Noun Phrase interpretation and type-shifting principles. J. Groenendijk, D. de Jongh, and M. Stokhof (eds), Studies in Discourse Representation Theory and the Theory of Generalized Quantifiers, 115-143. Dordrecht: Foris.

van Riemsdijk, H. 2005. Free relatives: A syntactic case study. The Blackwell Companion of Syntax, M. Everaert and H. van Riemsdijk (eds). Oxford, UK: Blackwell. 